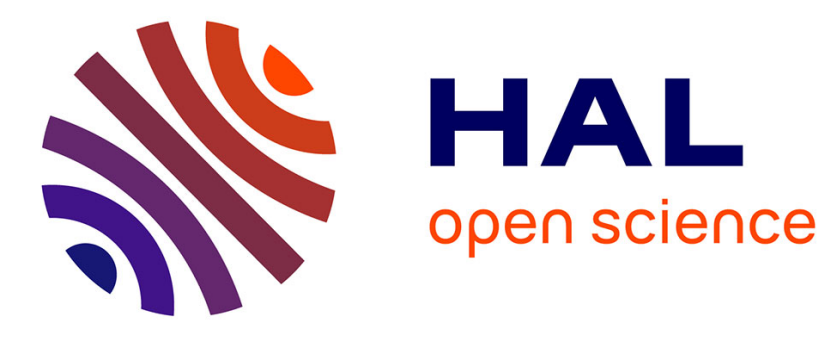

\title{
Les catégorisations enfantines de la politique
}

Alice Simon

\section{To cite this version:}

Alice Simon. Les catégorisations enfantines de la politique. Politiques de communication, 2018, Politiques de catégorisation du monde social, 10 (1), pp.193-223. 10.3917/pdc.010.0193 . hal-02297148

\section{HAL Id: hal-02297148 \\ https://hal.science/hal-02297148}

Submitted on 26 Sep 2019

HAL is a multi-disciplinary open access archive for the deposit and dissemination of scientific research documents, whether they are published or not. The documents may come from teaching and research institutions in France or abroad, or from public or private research centers.
L'archive ouverte pluridisciplinaire HAL, est destinée au dépôt et à la diffusion de documents scientifiques de niveau recherche, publiés ou non, émanant des établissements d'enseignement et de recherche français ou étrangers, des laboratoires publics ou privés. 
Alice Simon - Université de Montpellier, CEPEL

alicesimon@hotmail.fr

Les catégorisations enfantines de la politique

Résumé : À travers l'analyse des catégories que les enfants mobilisent pour décrire et juger l'univers politique spécialisé, cet article interroge le processus de construction des opinions politiques. Il montre que les opérations de catégorisation permettent à la fois aux enfants de comprendre le monde politique et de le juger : informations et jugements de valeurs sont souvent indissociables. Ce constat implique que les enfants intériorisent les jugements sur le monde politique comme étant des vérités indéniables sur le monde social, au même titre que les informations factuelles. L'article montre d'autre part que les catégories dont les enfants disposent pour comprendre et juger l'univers politique dépendent de leur compétence politique, laquelle varie notamment en fonction de leur milieu social. Les opinions des enfants peuvent ainsi reposer sur des systèmes de catégorisation assez sommaires (notamment l'opposition entre les "gentils" et les "méchants"), lesquelles sont parfois assorties d'arguments qui permettent aux enfants de justifier leur point de vue (en particulier à travers la catégorie "raciste"). Certains enfants, parmi les plus compétents, mobilisent également le clivage gauche-droite, lequel permet - et indique - une compréhension relativement élaborée du monde politique. Les catégorisations mobilisées par les enfants pour se repérer dans l'univers politique ne se valent donc pas dans la capacité qu'elles leur confèrent à asseoir et justifier leurs opinions.

Abstract: In order to investigate the opinion-making process, this article studies the categories that children use for describing and judging the specialized political universe. It shows that categorizations unable children both to understand and judge the political universe: information and judgments are inseparable. This implies that children take judgments about politics as incontestable truth, just as if they were factual information. The article also shows that the categories that children use in order to understand and judge the political universe depend on their political competence, which varies according to their social background. Children's political opinions can rely on very simple categories (especially the opposition between "good" and "bad" people), which are sometimes accompanied by some arguments (in particular the "racism" category). Some of the most competent children also use the right-left opposition, which is both a sign of and a tool for a sophisticated understanding of the political world. The categorizations that children use in order to understand politics are thus unequal, because they give them unequal ability to justify and assert their opinions.

Mots clefs : enfants ; enfance ; socialisation politique ; opinions politiques ; compétence politique.

Key words: children ; childhood ; political socialization ; political opinions ; political competence. 
Que les enfants puissent avoir des opinions politiques est une idée qui peut paraître absurde, voire dérangeante - en témoignent les difficultés rencontrées par les chercheurs interrogeant les enfants à ce sujet (Percheron, 1974 ; Tournier, 1997 ; Simon, 2017). Pour cause, les enfants sont jugés trop incompétents et trop immatures pour avoir leur propre opinion sur le monde politique : tout au mieux peuvent-ils «répéter » l'opinion de leurs parents et ainsi la révéler contre leur gré. Socialement indésirables, les opinions politiques des enfants sont également scientifiquement suspectes : les enfants sont considérés comme des enquêtés peu fiables (Kolson \& Green, 1970), dont les réponses sont particulièrement instables dans le temps (Vaillancourt ,1973). Ces difficultés méthodologiques ont participé à discréditer les travaux fondateurs sur la socialisation politique menés aux Etats-Unis dans les années 1960 et 1970 : postulant la durabilité des opinions acquises pendant l'enfance sans parvenir à la démontrer, ces travaux se sont heurtés à une impasse théorique qui a remis en cause l'utilité d'étudier les opinions des enfants. La science politique a ainsi cessé de s'intéresser aux enfants pendant plusieurs décennies - exception faite des travaux d'A. Percheron (1993) et de R.W Connell (1970). Pourtant, étudier les enfants peut nous renseigner sur la sociogenèse des comportements politiques. Dans la lignée des travaux récents réinvestissant l'étude des enfants en science politique (Throssell, 2015 ; Pagis \& Lignier, 2017b), il s'agit de centrer l'analyse sur les représentations des enfants, en laissant de côté non seulement la question de la durabilité des dispositions observées, mais aussi celle de leur origine - c'est-à-dire de l'influence des agents socialisateurs. Cette contribution part ainsi de l'hypothèse selon laquelle le rapport des enfants à la politique n'est pas par essence différent de celui des adultes, et qu'il peut donc être étudié à l'aune des concepts élaborés pour ces derniers. Si les enfants sont souvent peu compétents, se sentent souvent peu légitimes politiquement et sont perçus comme tels, ces caractéristiques rappellent celles des certaines fractions particulièrement peu politisées de la population adulte (Bourdieu, 1977 ; Gaxie, 1978). Partir de ce postulat permet non seulement d'utiliser la riche littérature sur les rapports des adultes à la politique pour comprendre ce que l'on observe chez les enfants, mais aussi de discuter cette littérature à partir d'une population d'enquête rarement étudiée par les politistes. Investiguer les rapports enfantins à la politique peut ainsi permettre non seulement de mieux connaître cette fraction spécifique de la population, mais aussi de contribuer à la compréhension des rapports ordinaires à la politique. Il s'agit en d'autres termes de désingulariser l'étude de l'enfance en général et des rapports enfantins à la politique en particulier.

Comment des individus objectivement et subjectivement peu compétents tels que les enfants parviennent-ils à défendre des opinions politiques? Cette problématique inscrit l'article dans la continuité de la littérature sur les outils à la disposition des citoyens, même les moins informés, pour former des opinions politiques. Cette littérature prend racine dans les travaux dits « cognitivistes » aux États-Unis, qui, faisant le constat que même les individus les moins politisés étaient en mesure de voter, ont cherché à montrer que tous les citoyens pouvaient avoir des opinions politiques grâce à l'usage de raccourcis ou encore d'heuristiques de jugements (Ferejohn \& Kuklinski, 1990 ; Popkin, 1991 ; Sniderman, Brody \& Tetlock, 1991). Les auteurs considèrent que les citoyens n'ont pas besoin d'avoir d'importantes connaissances politiques pour faire leur choix, et critiquent ceux qu'ils appellent les « minimalistes », notamment P. Converse (1964) à qui ils reprochent d'étudier les rapports à 
la politique sous le prisme exclusif des connaissances. Les cognitivistes ont alors ouvert tout un champ de recherche sur la façon dont les citoyens procédaient pour comprendre la politique, s'y repérer et former leurs opinions. Ils ont été critiqués par la suite - notamment en France par les héritiers de la sociologie bourdieusienne - parce qu'ils tendent à occulter le poids des différences sociales en considérant que tous les citoyens font usage d'heuristiques qui leur confèrent une égale capacité à opiner (Blondiaux, 2007 ; Gaxie, Hertaux \& Taiclet, 2016). Des travaux plus récents ont alors intégré les acquis des cognitivistes concernant les processus de construction de l'opinion, tout en étant attentifs aux inégalités de compétence qui impactent ce processus. Un nouveau champ de recherche décortique alors la façon dont les citoyens, en fonction du stock de connaissances à leur disposition, bricolent pour se faire une idée du monde politique et de ses clivages afin de pouvoir s'y repérer (Tiberj, 2004 ; Gaxie, 2007), font usage de schèmes plus ou moins élaborés qui les guide dans leur compréhension du politique (Joignant, 2004 ; 2007), se repèrent à travers un système de valeur (Sniderman, 1998), s'appuient sur des formes de traduction (Deloye, 2007), etc. Comme le résume D. Gaxie :

«On voit ainsi que tous les "citoyens", des moins concernés aux plus politisés, disposent d'instruments qui leur donnent une certaine "prise" sur des objets politiques et leur permettent de se prononcer. Mais on peut également constater que ces instruments sont de nature différente et, surtout, qu'ils ne sont pas équivalents du point de vue des capacités que des "citoyens" peuvent mettre en ouvre pour se faire une opinion sur les affaires de la cité et contrôler l'action des gouvernants » (2007, p.748).

Ces outils d'analyse n'ont à ce jour été que très peu mobilisés pour étudier le rapport des enfants à la politique. Cet article a pour ambition de montrer l'intérêt d'une telle approche, qui permet de renouveler l'étude du rapport des enfants à la politique et de formuler quelques hypothèses quant à la genèse des opinions politiques. Ces dernières sont ici comprises comme des prises de position qui reposent sur des dispositions. Elles peuvent être plus ou moins explicites (c'est-à-dire justifiables par des arguments rationnels), fermes (durables) et cohérentes avec les autres opinions de l'individu - elles se rapprochent donc plus ou moins de la forme idéal-typique de l'opinion solide, qui correspond au modèle socialement légitime de l'opinion politique. Plutôt que de fixer un seuil à partir duquel une prise de position est considérée comme suffisamment élaborée pour être nommée «opinion », je considère ici que toute prise de position - même sommaire - exprimant un goût, une préférence ou une croyance relève de l'opinion politique. Cette définition extensible des opinions politiques permet de repérer les multiples formes qu'elles peuvent prendre notamment en fonction du stock de connaissance dont disposent les individus.

L'article montre ainsi qu'à l'instar des adultes, les enfants peuvent avoir des opinions même lorsqu'ils disposent de très peu d'informations pour les défendre. Les jugements des enfants reposent en effet sur des opérations de catégorisations, qui leur permettent non seulement de situer les personnalités politiques mais aussi de se situer eux-mêmes vis-à-vis d'elles. Ces opérations de catégorisation font partie intégrante de la compréhension qu'ont les enfants de l'univers politique : elles constituent des outils à la fois cognitifs et normatifs, qui leur permettent de donner $d u$ sens à la politique. Les compréhensions de la politique reposent 
sur des catégorisations plus ou moins sophistiquées, qui confèrent aux enfants une inégale capacité à produire des discours politiques élaborés. Ces catégorisations peuvent ainsi être très simples, et se résumer à l'opposition gentil/méchant ou bien/mal (partie 1). Dans d'autres cas, elles sont assorties d'arguments politiques, notamment grâce à l'usage de la catégorie du racisme (partie 2), ou encore du clivage gauche/droite (partie 3), lequel n'est cependant accessible qu'aux enfants les plus compétents politiquement. Ainsi, si même les enfants les moins informés sont susceptibles d'émettre des opinions politiques, ils disposent d'éléments inégaux pour le faire : le degré d'élaboration des opinions politiques des enfants découle en grande partie de leur compétence - laquelle dépend de leurs propriétés sociales.

\section{Encadré méthodologique :}

Cet article est issu de l'enquête réalisée pour ma thèse, de 2014 à 2016, auprès d'élèves de CE2-CM1-CM2 d'une grande ville de province. L'enquête a eu lieu dans huit écoles aux profils diversifiés (écoles publiques, privées ou associatives; situées dans des quartiers favorisés, mixtes ou défavorisés; de petite, moyenne ou grande taille). Elle a consisté en principalement deux étapes :

- 1. Un questionnaire a été rempli par 538 enfants, de façon autonome pendant le temps scolaire. Le questionnaire comportait des questions sociodémographiques, un test de connaissance politique et des questions d'opinions. Parmi ces dernières, je fais référence dans cet article aux items suivants :

\section{Que penses-tu de François Hollande?}

qje l'adore $\quad$ je l'aime bien $\quad$ je ne l'aime pas $\quad$ je le déteste $\quad$ je ne sais pas $\quad$ je ne le connais pas

Pourquoi?

\section{Que penses-tu de Marine Le Pen ?}

qje l'adore $\quad$ je l'aime bien $\quad$ je ne l'aime pas $\quad$ je la déteste $\quad$ je ne sais pas $\quad$ je ne le connais pas

Pourquoi?

\section{Que penses-tu de Nicolas Sarkozy ?}

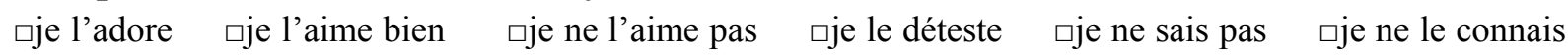
pas

Pourquoi?

- 2. Vingt-sept entretiens par binômes ont été menés avec cinquante-quatre enfants, auxquels s'ajoutent trois entretiens menés avec des groupes de quatre enfants. La méthodologie de l'entretien a été adaptée au public enfantin : j'ai utilisé un jeu sous forme de cartes plastifiées de différentes couleurs sur lesquelles étaient imprimées des questions, que les enfants piochaient au hasard. Ils devaient ensuite lire la question et y répondre : je n'intervenais que pour demander des précisions ou répéter les consignes ${ }^{1}$. Les cartes auxquelles je fais référence dans cet article sont les suivantes : la carte-question «la gauche et la droite, qu'est-ce que c'est ? ; la carte-concept «le racisme » (les enfants étaient invités à en expliquer le sens et

\footnotetext{
${ }^{1}$ Pour une analyse plus approfondie des spécificités méthodologiques rencontrées auprès des publics enfantins et des solutions que j'ai choisies pour y remédier, se référer à : Simon 2015.
} 
ce qu'ils en pensaient) ; les cartes-images de François Hollande, Nicolas Sarkozy et Marine Le Pen (les enfants devaient dire de qui il s'agissait et ce qu'ils en pensaient).

Interroger les enfants sur l'univers politique spécialisé constitue clairement une imposition de problématique, en tout cas pour la majorité d'entre eux. L'enquête, menée dans l'enceinte scolaire, constitue de plus une expérience inhabituelle pour les enfants. Les résultats analysés ici sont donc le produit de cette configuration discursive particulière, c'està-dire d'une relation adulte-enfant doublée d'une relation enquêteur-enquêté. Cette relation sociale produit des interactions qui ne sont ni plus, ni moins «vraies » que les relations sociales « ordinaires » (Mauger, 1991; Merklé \& Octobre, 2015). Elles révèlent ce qu'il se passe lorsque les enfants sont amenés à expliciter leur rapport à l'univers politique face à une étrangère et à un.e de leur camarade de classe - situation qui peut être inédite pour eux mais qui n'en demeure pas moins féconde pour l'analyse.

\section{Gentil/méchant : les premiers jalons de l'opinion}

$63 \%$ des enfants interrogés ont pris position dans le questionnaire au sujet de François Hollande (en cochant «je l'adore», «je l'aime bien», «je ne l'aime pas » ou «je le déteste »), ainsi que 62\% au sujet de Marine le Pen et 59\% au sujet de Nicolas Sarkozy. Les enfants qui connaissent a minima ces personnalités ${ }^{2}$ sont donc enclins à avoir - et livrer - une opinion à leur sujet. En effet, les connaissances sur l'univers politique sont rarement neutres : elles s'accompagnent au contraire de jugements de valeur, qui sont indissociables des informations acquises par les enfants sur l'univers politique. Les premiers jalons de la compréhension de l'univers politique sont ainsi également les premiers jalons de la construction de l'opinion : il s'agit pour les enfants de catégoriser les personnalités dans le camp du «bien » ou du «mal », des « gentils» ou des «méchants ».

\section{L'opinion peut se passer de raisons}

Lorsqu'on interroge les enfants au sujet de la politique spécialisée, un des phénomènes les plus étonnants est leur tendance à avoir des opinions sur des sujets qu'ils ne maîtrisent pourtant que très peu. F. Greenstein explique par exemple que « les sentiments, les évaluations et les affections politiques se forment bien avant que les enfants apprennent les informations pertinentes pour les justifier* » (1965, p.72). Plus récemment, W. Lignier et J. Pagis constatent que «les enfants savent d'abord ce qu'il faut penser politiquement avant de savoir pourquoi »(2017b, p.274). J'étaye ici ce constat et je propose d'en tirer des conclusions vis-à-vis de la construction de l'opinion. Nombre d'enquêtés expriment ainsi des avis tranchés sur les personnalités politiques, sans pour autant être en mesure de les justifier, dans le questionnaire comme lors des entretiens :

\footnotetext{
2 90\% des enfants ont reconnu François Hollande sur une photographie, contre $80 \%$ pour Nicolas Sarkozy et 77\% pour Marine Le Pen (Simon 2017, p.172).
} 
- Aurore $^{3}$ : [à propos de Nicolas Sarkozy] C'était notre ancien président.

- Ok, et qu'en penses-tu?

- Moi j'l'aime pas DU TOUT! [sur un ton catégorique]

- Pourquoi?

- Parce que je l'aime pas trop ... j'sais pas pourquoi mais moi j'l'aime pas trop. Moi je préfère celui-là [désignant la carte-image de François Hollande].

- Tu sais pas pourquoi ? [elle hoche la tête] T'as pas une idée ?[elle hoche la tête à nouveau] Tu penses que c'était un mauvais Président?

- oui !

[CE2, 06/2014. Parents travaillent «dans un bureau ». Bonne élève $]^{4}$

L'incapacité des enfants à justifier leur point de vue peut donner l'impression que celui-ci est livré «au hasard » et qu'il ne constitue donc pas vraiment une opinion - dans le sens où il ne reposerait pas sur une disposition mais sur une improvisation. Cette contribution défend au contraire l'idée que ces prises de position sont révélatrices de la façon dont se forme l'opinion, c'est-à-dire à travers un mécanisme d'intériorisation - d'un ordre moral, de valeurs et de croyances - et non d'un raisonnement supposant des informations et des arguments. Ainsi lorsque les enfants apprennent qui est par exemple Nicolas Sarkozy, ils apprennent par la même occasion si celui-ci est situé du côté de ceux qu'il faut aimer ou non, sans forcément obtenir - ou retenir - davantage d'informations à ce sujet. Les enfants mobilisent donc ce que la littérature anglo-saxonne appelle des heuristiques de jugement, opérations mentales qui permettent de produire des jugements même lorsque les informations sont limitées (Popkin, 1991 ; Mondak, 1993).

- Karim : [tire la carte-image de Nicolas Sarkozy] Ihhhhh ! [cri de dégoût] Je déteste ce gars !

- Yacine : Nicolas Sarkozy je l'aime bien ! Il me fait rigoler...

- Karim : J'l'aime pas !!!

- Pourquoi?

- [silence] Parce que ... parce que... j'ai oublié ! C'est parce qu'en fait... j'sais pas... j'sais pas. J'ai oublié. J'ai oublié.

- [à Yacine] Toi qu'est-ce que tu dis?

- Yacine : Bah moi je l'aime bien parce que j'aime bien son caractère.

- Qu'est-ce qu'il a son caractère?

- J'sais pas, j'aime bien!

[CM2, 06/2014. Karim : père maçon au chômage, mère au foyer. Bon élève. Yacine : père commerçant, mère couturière. Élève en difficulté.]

Bien que Karim ait «oublié » pourquoi il « déteste » Nicolas Sarkozy, cette déclaration n'en est pas moins de l'ordre de l'opinion politique, dans le sens où lui-même est convaincu de sa position, qui est par ailleurs accompagnée d'une charge émotionnelle, comme le montre sa réaction spontanée de dégoût à la découverte de la carte-image. Il s'agit donc d'une opinion solidement constituée, justement parce qu'elle ne s'appuie pas sur un argumentaire, mais sur

\footnotetext{
${ }^{3}$ Les prénoms d'origine ont été remplacés par des prénoms comparables du point de vue de leur origine géographique et/ou religieuse et de leur distribution sociale.

${ }^{4}$ Après chaque extrait d'entretien, je renseigne : la classe des enfants, la date de l'entretien, le métier des parents (renseigné par les enfants), le niveau scolaire (renseigné par les enseignant - information parfois manquante).
} 
une certitude quant à l'ordre des choses. Comme Karim, Youssef déclare dans l'extrait cidessous avoir «oublié » les raisons de son opinion. Cette explication semble tout à fait probable: les enfants ont pu disposer de plusieurs informations sur les personnalités politiques, qui les ont conduits à former une opinion à leur sujet. Bien qu'ayant oublié les informations sur lesquelles reposent leurs opinions, ils se rappellent de l'essentiel : si cette personnalité doit être « aimée » ou non.

- Youssef: Hé !! [Expression de bonne surprise] Bonjour mon pote François Hollande !

- Alors, qu'est-ce que t'en penses?

- Youssef : Il est cool, il est super cool, je l'aime bien.

- Pourquoi?

- Hum... parce qu'il dit des bonnes choses, sur la France. Il dit des bonnes choses, mais j'ai oublié sur quoi. Mon père aussi il l'aimait [son père est récemment décédé]. Ah... [ton nostalgique] il l'aimait beaucoup mon père aussi. Quand il était élu Président, il appelait tout le monde en disant... Non mais t'inquiète pas il fait toujours ça. Une fois quand on avait plus d'électricité il a même appelé le secrétaire de Fillon !

- Ton père il a appelé le secrétaire de François Fillon?

- Ouais, quand on avait plus d'électricité un jour!

- Et alors, ça a marché ?

- Euh... il a dit ok! juste ok... en tout cas François Hollande lui je l'aime bien, parce qu'il dit des bonnes choses...

- Et toi ? [à Medhi]

- Medhi : Bah moi aussi hein... pas pour des grandes choses hein, c'est bon, normal.

- Youssef [en même temps] : En tout cas j'étais content qu'il est devenu Président.

- [à Medhi] Pas pour des grandes choses?

- Medhi : Normal! Juste pour éviter Nicolas Sarkozy parce que j’aimais pas...

- Pourquoi?

- J'aime pas Nicolas Sarkozy!

- Youssef : Ouais c'est vrai que dans les rues là ils disaient «nique Sarkozy ! vers le

B. [nom d'un quartier proche de l'école].

[CM2, 05/2014. Youssef : père éboueur (décédé), mère au chômage. Élève en difficulté. Mehdi : père peintre en bâtiment, mère auxiliaire de vie. Élève moyen]

Il n'est donc pas nécessaire d'avoir des raisons pour avoir une opinion. « Aimer » ou « ne pas aimer » une personnalité politique ne dépend pas d'un raisonnement rationnel pesant le pour et le contre de ses positions programmatiques: il s'agit au contraire d'une évidence, intériorisée comme telle, qui peut se passer de justifications. L'opinion relève de ce point de vue d'une forme d'apprentissage, dans le sens où les enfants « apprennent » s'ils doivent ou non aimer une personne, en fonction de ce qu'ils entendent dans leur entourage. Les enfants citent d'ailleurs parfois leurs parents pour justifier leurs prises de position, à l'instar de Youssef dans l'extrait ci-dessus qui évoque son père, ainsi que des propos entendus dans le quartier. Doit-on considérer qu'il ne s'agit alors que de psittacisme, et qu'on n'est pas en présence d'une opinion «personnelle»? Toute opinion, y compris chez les adultes, se construit vis-à-vis du champ des opinions des autres, que celles-ci soient apprises par 
l'entourage ou par les médias ${ }^{5}$. En d'autres termes, l'opinion n'est jamais vraiment «personnelle ». Les opinions justifiées par la référence aux parents n'en sont pas moins des opinions : elles indiquent simplement que les enfants ne disposent pas des «instruments » nécessaires pour se les approprier et pour les défendre en leur propre nom. Il convient alors de distinguer le mode de justification de l'opinion de son «mode de production » (Bourdieu, 1979).

\section{Différencier mode de production et mode de justification de l'opinion}

Comme nous venons de le voir, les prises de position se résument souvent à la catégorisation «j'aime»/ «j'aime pas », parfois assortie de justifications plus ou moins élaborées : comme il était systématiquement demandé aux enfants, dans le questionnaire comme dans les entretiens, de se justifier (le plus souvent à travers la question «pourquoi ? »), ceux dont l'opinion n'a pas vraiment de raisons ont régulièrement improvisé une réponse - laquelle, bien que superficielle, nous renseigne sur les représentations des enfants sur les qualités attendues des personnalités politiques. Dans le questionnaire, les enfants justifient souvent leur opinion par des réponses non-argumentatives, presque tautologiques, par exemple «parce que c'est un bon président ${ }^{6}$, «il est bien », « il dirige pas très bien », « il fait bien son métier », «il est nul ! ». De la même manière, les personnalités politiques peuvent être qualifiées de « gentilles » (le plus souvent) ou parfois «méchantes ». Ces réponses montrent à quel point les opinions vis-à-vis des personnalités politiques peuvent relever de l'évidence: François Hollande est par exemple considéré comme «un bon président », Marine Le Pen est «méchante», et ces affirmations sont perçues comme des vérités sur le monde social plutôt que comme des prises de position qui nécessitent une argumentation. Cela ne signifie pas pour autant que les catégorisations des personnalités en «gentilles » ou «méchantes » soient toujours un artefact d'enquête : il semble au contraire que les enfants aient profondément intériorisé ces jugements sur le monde politique, qui ne s'accompagnent pas toujours d'explications supplémentaires.

- c'est quoi la différence entre la droite et la gauche?

- Atman : bah la droite c'est... nan j'sais pas... c'est méchant non?

- Tarek : extrême droite c'est les gentils et extrême gauche c'est les méchants !?

- Elyes [s'exclame] : beh c'est l'inverse !!

$[\ldots]$

- [à propos de François Hollande] qu'est-ce que vous en pensez?

- Elyes : gentil!

- Tarek : de François Hollande ? bah des fois il dit des choses bien des fois il dit des choses pas bien, parce que on a pas...

\footnotetext{
${ }^{5}$ Je suis ici le raisonnement de D. Gaxie (2013) : «On constate que les opinions émises par les membres des catégories les plus informées et mobilisées du public consistent le plus souvent en la reproduction d'arguments qui circulent dans les milieux politiques et journalistiques. [...] Les « amateurs » les plus compétents disposent surtout d'instruments de reproduction d'opinions. Ils sont souvent davantage reproducteurs que producteurs d'opinion, mais c'est en première personne qu'ils sont plus ou moins en mesure de s'approprier, de mémoriser et de mobiliser les prises de position qui circulent dans divers lieux de production et de diffusion idéologiques ».

${ }^{6}$ Ces réponses sont issues de questionnaires remplis de façon autonome par les enfants. Les fautes d'orthographe, très nombreuses, ont été corrigées pour faciliter la lecture.
} 
- Atman : [coupant] il a dit que ça a rien à voir avec les musulmans [à propos des attentats de Charlie Hebdo]

- Elyes : et moi je dis que c'est un bon président, c'est mieux que Barack Obama ou Marine le Pen.

[CM2, 01/2015.Tarek : mère au foyer, père travaille dans une maison de retraite. Elyes : mère aide à domicile, père vendeur de voitures. Atman : mère au foyer, père maçon]

Les réponses du type «il est bien », «elle est gentille » etc. ne sont donc pas uniquement des réponses de convenance: elles sont également significatives de la perception qu'ont ces enfants du monde politique. Beaucoup d'enfants justifient de plus leurs prises de position par des considérations générales sur ce que «font» ou «ne font pas » les personnalités politiques (par exemple «il fait beaucoup de choses »; «il fait aucune loi »; « il prenait de bonnes décisions »; « il fait rien »; « elle gère bien »; etc.), autant d'arguments qui permettent de justifier la catégorisation des personnalités dans la catégorie des «gentils » ou dans celle des «méchants », bien que les enfants ne disposent pas d'informations précises : ces réponses décrivent ce que doit être un bon président à leurs yeux. D'autres enfants invoquent les qualités esthétiques des personnalités («parce qu'il est rigolo avec ses lunettes »; «j'aime pas sa tête »; «il est de droite et porte des talons »; etc.), ou encore leurs qualités morales («il ment»; «il est un homme sincère et qui dit vrai »; «elle est inculte, dit des gros mots »; «il ne respecte pas les lois »; etc.). Les enfants peuvent donc mobiliser toute une panoplie d'arguments qui ne sont pas directement issus du champ politique pour juger celuici. Ils « recyclent» notamment des catégories de jugement provenant de leur famille, de leur école et même de leur groupe de pair (Lignier \& Pagis, 2017b, p.183), par exemple l'injonction d'être poli, de bien parler, ou encore de ne pas mentir - autant de critères à leur disposition pour produire une justification y compris en l'absence d'arguments politiques. L'apparence physique des candidats peut ainsi apparaître comme un mode spécifique de justification des opinions politiques, en particulier lors des entretiens, notamment parce que les discussions sur les personnalités politiques portaient sur des photographies :

- [à propos de François Hollande] c'est un bon Président à votre avis?

- Thelma : Oui

- Pourquoi?

- Ça se voit sur son visage!

- Ça se voit sur son visage que c'est un bon Président?

- Il a l'air gentil!

[CM2, 06/2014. Père entrepreneur en maçonnerie, mère au foyer. Bonne élève.]

On pourrait penser au premier abord que Thelma produit son opinion «sur le tas » en évaluant la photographie de François Hollande. Cette lecture serait en réalité simpliste : Thelma dira plus tard dans l'entretien que la droite et la gauche désignent «deux sortes de personnes, comme François Hollande j'pense qu'il est avec la gauche, Marine le Pen elle est avec la droite... la gauche c'est pour ceux qui vont aider les personnes... j'dis pas que la droite ça va pas aider les personnes mais... la gauche c'est un peu plus gentil j'pense ». Il est donc important de différencier le mode de production de l'opinion du mode de justification - ici, l'opinion de Thelma sur François Hollande semble bien plus politique que la justification 
qu'elle invoque spontanément pourrait le faire penser. Si la justification est produite « en mobilisant toutes les idées immédiatement accessibles » (Zaller, 1992, p.36), l'opinion semble avant tout découler d'une réappropriation de messages circulant dans l'environnement des enfants, qui les intériorisent comme des vérités indéniables. Comme le disent $\mathrm{P}$. Berger et $\mathrm{T}$. Luckmann, «l'enfant n'intériorise pas le monde de ses autrui significatifs comme un monde possible parmi beaucoup d'autres. Il l'intériorise comme le monde, le seul monde existant et concevable, le monde tout court » (Berger \& Luckmann, 2006 [1966], p.231).

Les enfants peuvent donc avoir des opinions politiques, y compris lorsqu'ils n'ont pas d'arguments pour les défendre. La catégorisation bien/mal leur suffit en effet pour se repérer dans l'univers politique et donc pour le juger. De nombreux enfants disposent cependant d'arguments qui leur permettent de justifier leurs opinions en mobilisant des catégories politiques - c'est-à-dire des catégories qui circulent effectivement dans l'univers politique. En premier lieu, les enfants peuvent s'appuyer sur une catégorie qui fait sens pour eux : le racisme.

\section{Le racisme : une catégorie politique accessible}

Ce qui distingue les «gentils » des «méchants » est parfois très explicite dans le discours des enfants. Les critères qu'ils mobilisent pour juger les personnalités politiques sont divers : certains évoquent par exemple l'écologie, d'autres l'égalité et la redistribution, d'autres encore la fermeté vis-à-vis du terrorisme. Mais la catégorie qui est - de loin - la plus fréquemment mobilisée par les enfants est celle du racisme : ce thème ressort dans la grande majorité des entretiens ( $a$ minima au sujet de Marine Le Pen), le monde politique étant même souvent compris au prisme de l'opposition entre les «racistes» et les autres. Pour une majorité d'enfants, cette catégorie est accessible cognitivement (ils en comprennent la signification et parviennent à l'utiliser à bon escient) notamment parce qu'elle est accessible dans leur environnement. La maîtrise de cette notion offre alors aux enfants une certaine compétence politique - souvent rudimentaire, mais qui leur permet de justifier leurs opinions en mobilisant une catégorie qui structure effectivement le champ politique.

\section{Une catégorie-repoussoir}

L'argument du racisme revient particulièrement en ce qui concerne Marine Le Pen : dans le questionnaire, 111 enfants, soit $55 \%$ de ceux qui ont donné une opinion à son sujet, l'accusent d'être raciste (en réponse à la question «pourquoi »). Les termes « raciste» ou « racisme » sont explicitement mentionnés dans la plupart de ces réponses : la réponse la plus fréquente est «elle est raciste ». De nombreux enfants donnent des détails supplémentaires, à l'instar de ces quelques exemples : «elle est raciste des Noirs et des Arabes »; « elle est raciste et de droite »; «sale raciste Marine Le Pen »; « elle est raciste !!!!! je la hais » etc. D'autres réponses ne mentionnent pas explicitement le terme « racisme » mais font mention de la politique migratoire imputée à Marine Le Pen : «elle veut enlever les Noirs et les Arabes »; «elle n'aime pas les musulmans »; «elle veut nous renvoyer au Maroc »; « il ne faut pas dire aux émigrés de partir »; etc. Bien que moins fréquemment, cet argument revient 
également régulièrement à propos des autres personnalités politiques, y compris dans les questionnaires : ainsi huit enfants disent ne pas aimer Nicolas Sarkozy «parce qu'il est raciste », «parce qu'il a dit un truc sur nous les Arabes », «même lui, raciste ! je le hais ». De plus, sept enfants utilisent ce critère pour juger François Hollande, qu'ils déclarent bien aimer «parce qu'il n'est pas raciste »; «parce qu'il a défendu les Marocains »; « lui il n'a pas dit "la France vous l'aimez ou vous la quittez" ». L'argument du racisme peut donc constituer un repère à partir duquel les enfants jaugent le monde politique : cette catégorie leur permet à la fois de situer les personnalités politiques les unes par rapport aux autres et de se situer euxmêmes.

Le racisme est en effet associé à une forte dépréciation morale. Une personnalité jugée « raciste » est systématiquement jugée «méchante»- la catégorie «raciste » est ainsi un argument à disposition des enfants pour justifier leurs opinions négatives, en particulier vis-àvis de Marine Le Pen. Pour cause, la norme sociale antiraciste est solidement ancrée chez les enfants rencontrés. Ainsi, lorsqu'ils piochent la carte «le racisme», tous les enfants qui connaissent ce terme précisent, en général spontanément, que «c'est mal », «c'est méchant », « ça sert à rien », etc. Le racisme relève ainsi du « savoir normatif » (Simon, 2017, p. 257), en ce sens qu'il s'agit d'une catégorie de pensée qui est à la frontière entre information et opinion: la dépréciation morale fait intégralement partie de la compréhension de ce terme. Notons d'ailleurs que plusieurs enfants ont raturé la photographie de Marine Le Pen dans le questionnaire ; deux enquêtés ont écrit des insultes en réponse à la question « qui est-ce »; un autre a barré la case «je la déteste» pour écrire à la place «je la hais ». Des réactions émotionnelles telles que des cris de dégoûts ont également été exprimées lors des entretiens, certains enquêtés demandant même à déchirer la carte-image ou à la cacher, en déclarant ne pas pouvoir supporter de la voir. L'accusation de racisme envers Marine Le Pen provoque donc un rejet très fort.

La charge normative du racisme en fait une catégorie opérante pour justifier les opinions politiques. Pour certains enfants, le racisme est d'ailleurs le principal critère à l'aune duquel ils évaluent le monde politique. On peut citer à cet égard le cas de Mehdi et Youssef, élèves de CM2 d'une école défavorisée de périphérie urbaine. Mehdi déclare, lorsqu'il tire la carte-question «quelles sont tes origines ? », «moi je suis algérien et je crois en Jésus », ce sur quoi Youssef répond «moi je suis guinéen et je crois en Allah ». Le père de Mehdi travaille dans le bâtiment et sa mère en maison de retraite. Le père de Youssef, récemment décédé, était «éboueur et grand voyant médium africain », sa mère est au chômage. Mehdi et Youssef font partie des enfants qui lisent la politique sous le prisme de l'opposition raciste / pas raciste : Mehdi dit à propos du clivage gauche/droite «j'ai déjà entendu ce mot ! C'est raciste et pas raciste ! Raciste et pas raciste !». L'ensemble des déclarations des enfants vont dans ce sens :

- [à propos de la carte-concept « le gouvernement »] Qu'en penses-tu?

- Youssef : Bof. Parce que des fois il nous met des lois mais... ces lois on les aime pas.

- Medhi [en même temps] : Des fois ils font n'importe quoi et des fois ils font des bonnes choses hein!

- Comme quoi?

- Medhi : Je sais pas. 
- Youssef : Parce que ils... les lois, des fois ils mettent des lois n'importe quoi. Pourquoi mes parents ils sont guinéens, pourquoi ils ont pas le droit de voter ? ils sont guinéens ils ont droit de voter...

- Medhi : [coupant] Oui c'est vrai ça! Moi j'aime pas Marine Le Pen c'est une raciste...

- Youssef : Ouais c'est... raciste, c'est... nan mais... quand je dis raciste ... mais c'est plus que raciste hein !

- Medhi : Mais ça m'énerve que... qu'il y ait du racisme parce que sinon... ce serait pas beau de voir que des Blancs que des Blancs, ou que des Noirs que des Noirs, ce serait pas beau!

- Youssef : Ouais en plus, pourquoi ils veulent nous faire partir, on les a fait quoi ! [s'énervant] Qu'est-ce qu'on les a fait, pourquoi on doit partir ? On les a fait quoi !

- Medhi : Nicolas Sarkozy encore moins... j'aime pas!

- Youssef : [en même temps] Alors lui il a voulu nous rajouter trois mois de grandes vacances!

- Medhi : Ouais mais ça va... on va pas glander glander...

- Youssef : Ouais mais trois mois de grandes vacances c'est cool hein !

- Medhi : Ouais mais... j'aime pas ses gestes...

- Quels gestes?

- Youssef : Racistes. Ouais c'est vrai en ce moment y'a beaucoup de racisme dans le gouvernement...

- Medhi : Hum [confirmant]. Dans la droite et la gauche.

- Youssef : Moi j'suis pour le côté François Hollande. Lui il est pas raciste.

[CM2, 05/2014. Youssef : père éboueur (décédé), mère au chômage. Élève en difficulté. Mehdi : père peintre en bâtiment, mère auxiliaire de vie. Élève moyen]

Cet échange a lieu quasiment au début de l'entretien : il est intéressant de constater que les enfants évoquent spontanément le thème du racisme, à l'aune duquel ils évaluent les trois personnalités politiques les plus connues des enfants, avant que je les aie interrogés à leur sujet. Le racisme agit donc ici comme une catégorie politique qui permet aux enfants de se repérer sur l'échiquier politique.

\section{L'usage différencié de la catégorie « raciste »}

Si la lecture du politique sous le prisme du racisme n'est pas propre aux enfants racisés $^{7}$, elle se retrouve de façon plus systématique chez ces derniers. Une analyse attentive des entretiens indique en fait que l'argument du racisme est mobilisé de façon différenciée selon les enfants, en fonction de leur sentiment d'appartenir ou non aux catégories qu'ils accusent les personnalités politiques de stigmatiser. Ainsi le «nous » (musulmans, Marocains, Arabes...) est présent dans de nombreuses réponses au questionnaire, qui proviennent toutes d'enfants déclarant des origines étrangères, par exemple «elle a dit que si elle est présidente elle renvoie les Algériens dans leur pays »; «parce qu'elle veut nous renvoyer »; « elle veut renvoyer les Marocains au Maroc »; "parce qu'elle est raciste et si elle devient présidente mon Papa sera exclu de la France »; «parce qu'il a dit un truc sur nous les Arabes ». Ces réponses témoignent donc du sentiment de ces enfants d'être personnellement concernés, en

\footnotetext{
${ }^{7}$ Ce terme fait référence au sentiment exprimé par les enquêtés d'appartenir à une catégorie raciale minorisée (Poiret, 2011 ; Lorcerie, 2003).
} 
tant que membres d'un «nous » racisé, par les propositions politiques jugées racistes. Les enfants qualifient ainsi généralement le racisme de Marine Le Pen en fonction de leur propre origine, évoquant «les Arabes », «les musulmans », voire «les Marocains » quand euxmêmes sont d'origine marocaine, «les Algériens » lorsqu'ils sont d'origine algérienne, etc. Ainsi lorsqu'il s'agit d'évoquer les idées jugées racistes de Marine Le Pen, les enfants rapportent fréquemment leurs opinions politiques à leur groupe d'appartenance, ce qui témoigne d'une forme d'ethnicisation des opinions politiques (Braconnier \& Dormagen, 2010). Pour ne citer qu'un seul extrait d'entretien, prenons celui mené juste après les attentats de Charlie Hebdo avec quatre élèves de CM2 de milieux populaires, se déclarant tous d'origine étrangère :

[Je montre la carte-image de Marine le Pen. Les enfants crient son nom à l'unisson, Tarek rit, Elyes fait un cri de dégoût.]

- Tarek : Moi j'l'aime pas.

$[\ldots]$

- Tarek : Moi j'vous dis pourquoi je l'aime pas, parce que elle avait dit une fois que... que les musulmans [Elyes : ils sortent de la France] oui, ils vont retourner dans leur pays. Parce que moi j'ai de la famille qui sont nés...

- Atman : [coupant] Elle veut nous renvoyer au Maroc.

- Elyes : Elle a pas dit les musulmans, elle a dit les...

- Atman [coupant] : Y'a que les Français qui viennent !

- Elyes : T'es bête toi t'es français, donc tu va rester sans tes parents !

- Tarek: Y'a que les Français qui viennent ici, moi j'aime pas parce que après, bah regardez le père d'Atman bah c'est un Marocain, et lui il est né en France, et après il va se séparer de son père!

- Atman : Moi j'vais avec lui, t'es ouf!

- Tarek: Bah voilà, elle a pas le droit de faire ça, parce qu'après on se sépare de ses enfants.

- Bader : Et après il vont pas reconnaître leurs parents, et quand il va grandir il va dire «non c'est pas toi ma mère arrête de te moquer » et après quand ils vont pas la croire...

- Elyes : [coupant] Non c'est un conte de fée ça !

- Tarek : Non mais parce que elle a dit «y'a que des Français qui ont le droit de rester en France, c'est tout » chacun dans son pays après elle s'occupe pas des autres pays, que de... voilà elle a dit ça.

[CM2, 01/2015.Tarek : mère au foyer, père travaille dans une maison de retraite. Elyes : mère aide à domicile, père vendeur de voitures. Atman : mère au foyer, père maçon. Bader : mère au foyer, père maçon.]

Les opinions de ces enfants à propos de Marine Le Pen sont donc directement liées à leur situation personnelle. Tarek, qui se dit «d'origine algérienne», évoque «les musulmans » puis «l'Algérie », tandis qu'Atman évoque exclusivement «le Maroc ». L'échange entre les enfants leur permet ensuite de monter en généralité pour évoquer une discrimination envers les non-Français en général, tout en mobilisant en exemple leur situation en particulier. Le scénario selon lequel Atman devrait se séparer de son père témoigne d'une inquiétude diffuse, largement fantasmée (comme le rappelle d'ailleurs Elyes : «c'est un conte de fée ça »!) qui montre en tout cas à quel point les opinions politiques sont liées au sentiment d'appartenir à une minorité raciale. 
Cela ne signifie pas pour autant que seuls les enfants racisés accusent Marine le Pen de racisme. Cet argument est au contraire très répandu, mais prend des formes différentes chez les enfants qui ne se sentent pas eux-mêmes concernés par le racisme en question. Premièrement, ceux-ci font beaucoup plus souvent référence aux « Noirs » qu'aux « Arabes », «musulmans», «Marocains» etc. qui sont plus fréquemment mentionnés par leurs camarades racisés $^{8}$. Les «Noirs » agissent ainsi comme figure archétypale de victimes du racisme. De nombreux enfants mentionnent par ailleurs «les étrangers » ou plus rarement «les immigrés », catégories qui ne sont quasiment jamais utilisées par les enfants racisés, qui évoquent éventuellement «ceux qui ne sont pas Français ». Pour autant, les enfants nonracisés peuvent eux aussi relier leurs opinions vis-à-vis du racisme à leur vie personnelle, mentionnant un «tonton qui est noir» et qui risquerait d'être "renvoyé », une «amie amérindienne » ou encore une camarade de classe «qui n'est pas française ». Les situations personnelles agissent donc comme des exemples qui permettent de donner du sens et conférer une dimension émotionnelle à la dépréciation de certaines personnalités politiques.

Pour synthétiser, le critère du racisme permet aux enfants de se repérer sur le champ politique en catégorisant les personnalités dans le camp des «gentils » ou des «méchants », à partir d'un critère qui fait sens pour eux. La saillance de cette catégorie dans les représentations enfantines de la politique est symptomatique de l'importance qu'a pris l'immigration dans les débats politiques, en particulier pour les populations qui se sentent personnellement visées par ces débats - au premier rang desquels les jeunes considérés comme «issus de l'immigration » (Barrault-Stella \& Berjaud, 2016). Il s'agit, de plus, d'un critère que les enfants peuvent se réapproprier facilement, non seulement parce qu'il est consonant avec les messages familiaux et scolaires mais aussi parce qu'il est associé à une dépréciation univoque. Le racisme est ainsi une catégorie politique accessible - à la fois parce qu'elle est fréquemment mobilisée dans l'entourage des enfants et parce qu'elle est facilement compréhensible. Tous les enfants sont donc susceptibles de la mobiliser - quoique de façon différenciée selon qu'ils se sentent appartenir ou non à un «nous » racisé. Le clivage gauchedroite, quant à lui, n'est accessible qu'à une minorité d'enfants, parmi les plus compétents du groupe étudié.

\section{Le clivage gauche/droite : un repère pour les plus compétents}

L'opposition raciste/pas raciste offre aux enfants une compréhension rudimentaire des enjeux qui structurent le champ politique - compréhension qui leur permet de se situer vis-àvis des personnalités qu'ils connaissent. De la même manière, le clivage gauche/droite agit comme un repère assorti d'un jugement de valeur. Si ces deux systèmes de catégorisation permettent aux enfants de se repérer et de situer dans l'univers politique, le clivage gauche/droite - qui n'est accessible qu'à une minorité d'enfants particulièrement compétents - est le signe d'une compréhension beaucoup plus élaborée du monde politique. Ainsi, disposer de systèmes de catégorisation pluriels et sophistiqués permet aux enfants de monter

\footnotetext{
${ }^{8}$ La ville où a eu lieu l'enquête a connu des vagues migratoires principalement issues des pays du Maghreb. Rares sont les enquêtés susceptibles de se considérer comme Noirs.
} 
en généralité et de produire des opinions politiques plus proches du modèle socialement légitime de l'opinion - c'est-à-dire une opinion justifiable par des arguments rationnels.

\section{Une catégorie inégalement maîtrisée}

Le clivage gauche-droite est une des principales catégories dont disposent les individus pour se repérer dans l'univers politique (Tiberj, 2004), a fortiori en France où ce clivage est un principe majeur de structuration de l'espace partisan (Le Bohec \& Le Digol, 2012) et un marqueur idéologique important pour les électeurs (Marcel \& Witowksi, 2003). Pour autant, tous les Français ne font pas le même usage de ces catégories : « une partie de la population, spécialement parmi les catégories à faible capital culturel, ne parvient ni ne cherche à se les approprier » (Sapiro, 2012, p.314). Les rares études menées sur les enfants à ce sujet confirment l'importance du clivage gauche-droite dans les représentations de la politique : les travaux d'A. Percheron (1980) indiquent que les enfants se repèrent davantage à partir du clivage gauche-droite qu'à partir du système partisan, contrairement aux enfants états-uniens. Plus récemment, les recherches de W. Lignier et J. Pagis (2017a) ont montré que ce clivage était très inégalement compris en fonction de l'âge, de l'origine sociale ou encore du genre des enfants. Les données issues de mon questionnaire confirment ce constat. Ainsi les enfants qui mobilisent spontanément ces catégories pour juger les personnalités dans le questionnaire sont très minoritaires : 19 enfants seulement mobilisent le terme « gauche » ou «droite » pour une des personnalités au moins (soit $7 \%$ des réponses rédigées). Notons que ces enfants tendent à juger les trois personnalités (pour 8/19 d'entre eux) ou au moins deux d'entre elles (7/19) à partir du clivage gauche-droite, ce qui indique que celui-ci agit comme une catégorie politique à partir de laquelle ils se repèrent concernant l'intégralité du champ. Les enfants disent par exemple aimer François Hollande «car il est de gauche »; «car je soutiens la gauche et j'aime bien ses idées », ou au contraire ne pas l'aimer car « [il est] de gauche mais il bascule sur la droite », ou car «il dit qu'il est au milieu mais en fait il est à droite ». De la même manière, Marine Le Pen est accusée d'être « de droite », « raciste et de droite », ainsi que Nicolas Sarkozy («je n'aime pas l'idée des candidats de droite », « il est du côté droit», etc.). Les enfants qui mobilisent le clivage gauche-droite dans le questionnaire le font ainsi toujours à bon escient, aucun ne se trompe sur le positionnement d'une personnalité. Il est intéressant de constater que tous les enfants qui mobilisent cette catégorie politique, sans exception, valorisent la gauche. De plus, tous ces enfants déclarent que leurs deux parents sont de gauche, sauf un enfant qui les déclare «ni à droite ni à gauche ». Le test de connaissance politique inclus dans le même questionnaire indique en effet que les enfants qui connaissent le clivage gauche-droite déclarent presque tous être issus de familles de gauche (Simon, 2017, pp.205-207). La communication familiale autour de cette notion semble alors être quasi exclusivement le fait de parents s'identifiant à la gauche. Les enfants qui mobilisent ce clivage ont par ailleurs un niveau plus élevé de connaissances politiques et ils sont quasiment systématiquement scolarisés dans les écoles favorisées. D'ailleurs, leurs parents sont le plus souvent issus des classes favorisées (10/19), voire des classe moyenne (8/19), et très rarement des classes populaires (1/19), pourtant légèrement surreprésentées dans l'échantillon. Cette distribution peut s'expliquer par la corrélation entre la compétence politique des enfants et leur milieu social (Simon, 2017, pp.195-199), corrélation qui apparaît 
cependant particulièrement forte pour cette notion spécifique. Ce que l'on observe n'est alors peut-être pas tant lié à un écart dans le degré de politisation des familles en fonction de leur milieu social qu'à une disparité dans les cultures politiques familiales, le clivage gauche-droite semblant avoir une place plus importante dans les milieux favorisés de gauche. Il est d'autre part intéressant de noter que l'âge ne détermine pas les chances de mobiliser le clivage gauche-droite (alors que l'âge a un impact majeur sur le niveau de connaissance politique des enfants, Simon, 2017, pp.188-191) : sept des dix-neuf enfants qui le mobilisent sont en CE2, soit un peu plus d'un tiers d'entre eux, ce qui correspond au pourcentage de CE2 parmi les enquêtés. Cela indique donc que les enfants qui sont socialisés à mobiliser ce clivage le sont dès le plus jeune âge : nous sommes bien en présence d'une différence liée à l'environnement social et non au développement cognitif. Ces propos ne concernent que les enfants qui choisissent spontanément de mobiliser le clivage gauche-droite pour justifier leur opinion dans le questionnaire. Beaucoup d'autres enfants connaissent ce clivage et sont susceptibles de le mobiliser lors des entretiens, sans pour autant en avoir fait le choix dans le questionnaire. Si leur profil social est un peu plus diversifié, les classes moyennes et supérieures restent largement surreprésentées - et la gauche est presque systématiquement préférée à la droite. Ces résultats confirment que l'origine sociale joue très fortement dans la façon dont les enfants perçoivent et jugent l'univers politique.

\section{L'expression d'une compétence politique}

La maîtrise du clivage gauche-droite va de pair avec une certaine compétence politique, non seulement parce que seuls les enfants les plus compétents le mobilisent, mais aussi parce qu'il leur permet de justifier efficacement leurs opinions politiques et donc de s'affirmer lors d'une discussion. En d'autres termes, la mobilisation de ce clivage est à la fois un indice de la compétence politique des enfants et un outil qui leur permet de la mettre en œuvre lors des entretiens. Les enfants associent en effet ces notions à d'autres systèmes de catégorisations qui leur permettent de donner du sens à ce clivage et d'avoir une opinion à son sujet. Ainsi lorsque les enfants connaissent le clivage gauche-droite, ils l'assortissent d'un jugement de valeur - la gauche étant, nous l'avons dit, quasiment systématiquement préférée à la droite. En particulier, les enfants associent souvent le clivage gauche-droite à l'opposition raciste / pas raciste, ce qui provoque une dépréciation univoque de la droite. Mais les perceptions enfantines du clivage gauche-droite dépassent souvent la seule opposition raciste / pas raciste, pour intégrer d'autres éléments d'explications, comme dans l'extrait ci-dessous :

- Kays : Bah la droite sont racistes, ils ... la droite veulent faire une bombe nucléaire, et aussi ils veulent que Marine le Pen soit la présidente, de la république.

- Amy : Ouais.

- Kays : Et la gauche ils veulent pas.

- Amy : [en même temps] La gauche ils sont très gentils.

[CE2, 02/2016. Kays : père animateur périscolaire et barman. Mère dans le milieu du handicap. Bon élève. Amy : mère animatrice-coordinatrice, père militaire. Bonne élève].

On voit donc que le clivage gauche-droite a du sens pour Kays, parce qu'il l'associe à d'autres informations : ce clivage lui permet non seulement de distinguer les "gentils" des 
"méchants", mais également d'expliquer pourquoi (en l'occurrence, parce que la droite est associée au racisme, à la bombe nucléaire et à Marine Le Pen). Cela signifie que derrière les termes "gauche" et "droite", sont sous-entendus plusieurs éléments qui sont le signe d'une appréciation relativement complexe de l'univers politique. Il est intéressant de constater que ces associations diffèrent en fonction des enfants : si le racisme est l'argument qui revient le plus souvent, d'autres enfants ont une lecture plus économique de ce clivage, à l'instar de Maël et Chloé.

- Maël : à gauche, les idées sont plutôt que le... que le monde ...

- Chloé : communiste, parfois... 'fin moi aussi je préfère... je préfère les idées de gauche, parce que y'a des idées franchement que je trouve bien mais voilà...

- qu'est-ce que t'allais dire? [à Maël]

- Maël : Beh de gauche c'est plutôt... ils veulent plutôt un monde plus... 'fin peut-être que je me trompe hein, mais plus... équilibré, 'fin qu'y'ait pas trop de pauvres, qu'ils soient tous équilibrés...

- Chloé : [...] Moi ce que j’aime bien c'est leur idée... l'écologie, le partage, tout ça... [CM2, 02/2016. Maël : père assistant réalisateur et comédien, mère tient un café associatif. Très bon élève. Chloé : père comédien et metteur en scène, mère comédienne et formatrice dans le théâtre. Bonne élève.]

Ainsi les enfants qui mobilisent le clivage gauche-droite lui associent des informations à la fois cognitives (qui expliquent ce qu'il signifie) et normatives (indissociables d'un jugement de valeur). Le clivage gauche-droite agit alors pour ces enfants comme un repère (Tiberj, 2004), qui leur permet de donner du sens au monde politique. Nous pouvons citer à cet égard l'échange avec Jeanne et Clothilde, élèves de CE2 de l'école Centre-Fav. Toutes deux sont issues de milieux favorisés intellectuels : le père de Jeanne est professeur des universités en sciences sociales, sa mère vendeuse dans une boutique de décorations. Le père de Clothilde est quant à lui architecte et sa mère urbaniste. Toutes deux sont de très bonnes élèves et détiennent un niveau de connaissance politique particulièrement élevé pour leur âge. Jeanne et Clothilde mobilisent des explications différentes pour décrire le clivage gauchedroite :

- Clothilde : [carte-question] «La gauche, la droite, qu'est-ce que c'est ? » ben... en fait et bah c'est... il peut avoir un maire qui est de gauche, et il fera pas les mêmes ... il fera pas le même genre de lois que un maire qui est de droite. Parce que de... les... ceux de droite ils sont un peu racistes, et... voilà.

- Jeanne : Ouais.

- Tu veux rajouter quelque chose? [à Jeanne]

- Euh en fait c'était... euh je crois que c'était au Moyen-âge qu'on avait les rois, et on les a remplacé par les présidents, la droite c'était ceux qui étaient pour les rois un peu, et la gauche c'était ceux qui voulaient plus avoir de roi.

Clothilde associe donc le clivage gauche-droite à l'opposition raciste - pas raciste, à laquelle Jeanne ajoute une explication historique, associant la gauche à la République et donc au progrès démocratique. Toutes deux, bien qu'à l'aise pendant l'entretien et assez loquaces au sujet de leur vie quotidienne, de leur école ou des questions de société, sont assez brèves 
lorsqu'il s'agit de juger les personnalités politiques. La catégorisation normative des personnalités repose ainsi principalement chez ces deux enquêtées sur leur affiliation à la gauche ou à la droite :

- Clothilde : [à propos de Marine Le Pen] Ben... comme j’suis pas trop droite ben j'l'aime pas trop, 'fin...

- Et toi Jeanne?

- Jeanne : Ben moi j'l'aime pas. Mais le pire j'trouve que c'est son père.

- pourquoi?

- Parce que. [Catégorique, puis reprend] C'est son père qui l'a élevée !

- Donc tu la préfères elle à son père?

- Pas de loin!

- Pas de loin [rires], et pourquoi tu l'aimes pas?

- Parce qu'elle est... elle est au Front National! Eh bah mes parents ils votent à gauche et tout. Moi j'peux pas voter.

- Clothilde : Non mais toi t'es de gauche ou de droite?

- Jeanne : Gauche !

- Et toi aussi?

- Clothilde : Oui ! [Jeanne prend une nouvelle carte]

Jeanne et Clothilde, contrairement à la majorité des enfants, n'évoquent pas l'argument du racisme a propos de Marine Le Pen : le clivage gauche-droite agit comme un repère plus général, car nous avons vu qu'il inclut, pour ces enquêtées, le critère du racisme. Il est d'ailleurs intéressant de noter que Clothilde pose directement la question à Jeanne « toi t'es de gauche ou de droite ? », pour que celle-ci confirme leur identification commune à la gauche - laquelle est présentée comme relevant de l'évidence. Pour Jeanne et Clothilde, la gauche et la droite constituent des repères qui leur permettent de situer les différentes personnalités politiques, non seulement dans le champ politique mais également dans leur propre hiérarchie normative. Le clivage gauche-droite leur permet de catégoriser efficacement les personnalités politiques en associant entre elles plusieurs informations (droite $=$ raciste et conservateur $=$ méchant). Incontournable dans le champ politique, cette catégorie offre donc aux enfants les plus compétents une compréhension relativement élaborée du monde politique - dans le sens où ils maîtrisent les enjeux qui structurent effectivement le champ politique ainsi que la terminologie légitime pour les désigner.

\section{Conclusion}

Les opinions personnelles se forment donc à travers des opérations de catégorisation, plus ou moins complexes en fonction des informations à disposition des enfants. Ainsi, la catégorisation «bien / mal» ou «gentil / méchant» constitue un premier principe d'ordonnancement symbolique du milieu politique, que les enfants peuvent mobiliser y compris en l'absence d'arguments supplémentaires. Les enfants disposent régulièrement d'éléments qui leur permettent de formuler des opinions politiques plus élaborées : le racisme apparaît notamment comme une catégorie accessible à une majorité d'enfants, qui leur permet de se repérer sur le champ politique et de justifier leurs opinions. Certains enfants, parmi les 
plus compétents, mobilisent également le clivage gauche-droite : celui-ci leur offre une compréhension plus fine du monde politique que le repère raciste/pas raciste, qui est d'ailleurs souvent inclus dans la compréhension de la catégorisation gauche/droite. Si les enfants peuvent donc formuler des opinions politiques à propos des personnalités qu'ils connaissent, y compris lorsqu'ils disposent de très peu d'informations à leur sujet, les repères dont ils disposent ne se valent donc pas dans la capacité qu'ils leur confèrent à comprendre le monde politique et à étayer leurs opinions par des arguments. La compétence politique est donc un facteur déterminant de la capacité des enfants à asseoir et justifier leurs opinions.

L'examen des catégorisations enfantines de la politique indique également que les jugements de valeur ont une place majeure dans les apprentissages politiques. La catégorisation est à la fois une opération cognitive, dans le sens où elle permet aux enfants de comprendre la politique, et une opération normative - qui leur permet de se situer. Ainsi, les informations et les jugements sur les personnalités politiques sont acquis de façon simultanée : savoirs et opinions découlent de la réappropriation de messages circulant dans l'environnement de l'enfant - principalement, si on en croit la littérature sur le sujet, dans sa famille (K. Jennings, L. Stoker \& J. Bowers, 2001 ; Muxel, 2001). Les enfants intériorisent ces messages comme étant des vérités indéniables, par exemple « Nicolas Sarkozy fait partie des méchants ». Au gré de l'acquisition d'informations sur le monde politique, cette première catégorisation pourra être étoffée par des éléments supplémentaires : «Nicolas Sarkozy fait partie des méchants parce qu'il est raciste », éventuellement «il est raciste parce qu'il est de droite ». Ce processus ne suit pas nécessairement un déroulement linéaire : les enfants peuvent oublier les justifications qui sous-tendent leurs opinions, et ils sont par ailleurs susceptibles de changer d'opinion si les messages provenant de leur environnement se modifient. Quoiqu'il en soit, cette étude indique que les enfants «apprennent» leurs opinions, davantage qu'ils ne les «construisent». Ce constat explique d'ailleurs pourquoi la transmission des opinions politiques fonctionne si bien: les parents n'ont pas besoin de convaincre leurs enfants à travers des arguments rationnels pour leur transmettre leurs opinions politiques, car les enfants intériorisent celles-ci comme étant de l'ordre de la vérité indéniable sur le monde et sur eux-mêmes.

\section{Références bibliographiques}

- Berger P., Luckmann T., La construction sociale de la réalité (1966), Armand Colin, 2012.

- Berrault-Stella L., Berjaud C., «"Celui qui parle de ça, limite on va le vanner" ; Des jeunes de classes populaires face aux élections de 2012 », Les sens du vote; Une enquête sociologique (France 2011 - 2014), sous la dir. Collectif Sociologie Politique des Elections, Presses Universitaires de Rennes, 2016.

- Blondiaux L., «Faut-il se débarrasser de la notion de compétence politique ? Retour critique sur un concept classique de la science politique », Revue française de science politique, 2007, 57(6).

- Bourdieu P., «Questions de politique », Actes de la recherche en sciences sociales, 1977, 16 , septembre.

- Bourdieu P., La distinction ; critique sociale du jugement, Editions de Minuit, 1979. 
- Braconnier C., Dormagen J.-Y., «Le vote des cités est-il structuré par un clivage ethnique?», Revue française de science politique, 2010, 60(4).

- Connell R.W., The Child's Construction of Politics, Melbourne University Press, 1971.

- Converse P., «The nature of belief systems in mass publics », Ideology and discontent, sous la dir. D. Apter, The New Press of Glencoe, 1964.

- Deloye Y., "Pour une sociologie politique de la compétence à opiner "politiquement" . Quelques hypothèses de travail à partir de l'histoire électorale française », Revue française de science politique, 2007, 6(57).

- Ferejohn J. A., Kuklinski J. H. (eds), Information and Democratic Processes, University of Illinois Press, 1990.

- Gaxie D., Le cens caché, Seuil, 1978.

- Gaxie D., «Cognitions, auto-habilitation et pouvoirs des « citoyens » », Revue française de science politique, 2007, 57 (6).

- Gaxie D., «Retour sur les modes de production des opinions politiques », Trente ans après La Distinction de Pierre Bourdieu, sous la dir. P. Coulangeon \& J. Duval, La Découverte, 2013.

- Gaxie D., Hertaux J., Taiclet A.-F., «"Nabot !" "Gros pouf !" ; Jauger, Juger la politique », Les sens du vote; Une enquête sociologique (France 2011 - 2014), sous la dir. Collectif Sociologie Politique des Elections, Presses Universitaires de Rennes, 2016.

- Greenstein F., Children and politics, Yale University Press, 1965.

- Jennings K., Stoker L., Bowers J., Politics Across Generations: Family Transmission Reexamined, Institute of Governmental Studies, 2001.

- Joignant A., « Pour une sociologie cognitive de la compétence politique », Politix, 2004, 17 (65).

- Joignant A., «Compétence politique et bricolage; les formes profanes du rapport à la politique », Revue Française de Science Politique, 2007, 57 (6).

- Kolson K., Green J., «Response Set Bias and Political Socialization Research », Social Science Quarterly, 1970, 51(3).

- Le Bohec J., Le Digol C. (dir.), Gauche/Droite. Genèse d'un clivage politique, Presses Universitaires de France, 2012.

- Lignier W., Pagis J., «"Left" vs. "Right": How Children Reconstruct the Political Field», American Behavioral Scientist, 2017a, 61(2).

- Lignier W., Pagis J., L'enfance de l'ordre ; comment les enfants perçoivent le monde social, Seuil, 2017b.

- Lorcerie F., L'école et le défi ethnique: éducation et intégration, ESF, 2003.

- Marcel S., Witkowski D., «Il faut sauver le clivage gauche-droite », L'état de l'opinion 2003, sous la dir. O. Duhamel \& P. Méchet, Seuil, 2003.

- Matefia A., «La lutte contre les discriminations, un rapport de domination ? Les jeunes des quartiers populaires au croisement des représentations», communication présentée aux journées d'étude "Penser l'intersectionnalité dans les recherches en éducation : enquêtes, terrains, théories », ESPE de l'académie de Créteil, 18 et 19 mai, 2017.

- Mauger G., «Enquêter en milieu populaire », Genèses, 1991, 6. 
- Mercklé P., Octobre S., «Les enquêtés mentent-ils ? Incohérences de réponse et illusion biographique dans une enquête longitudinale sur les loisirs des adolescents. », Revue française de sociologie, 2015, 56 (3).

- Mondak J., «Public Opinion and Heuristic Processing of Source Cues », Political Behavior, 1993, 15.

- Muxel A., «Les choix politiques des jeunes à l'épreuve du temps Une enquête longitudinale », Revue française de science politique, 2001, 51(3).

- Percheron A., L'univers politique des enfants, Armand Colin, 1974.

- Percheron A., L’élection du Président et les enfants, Pouvoirs, 1980, 14.

- Percheron A, la socialisation politique, Armand Colin, 1993.

- Poiret C., «Les processus d'ethnicisation et de raci(ali)sation dans la France contemporaine: Africains, Ultramarins et "Noirs"», Revue Européenne des Migrations Internationales, 2011, 27(1).

- Popkin S. L., The Reasoning Voter; Communication and Persuasion in Presidential Campaigns, The Chicago University Press, 1991.

- Sapiro G., "De l'usage des catégories de droite et de gauche dans le champ littéraire », Gauche-droite. Genèse d'un clivage politique, sous la dir. J. Le Bohec \& C. Le Digol, Presses Universitaires de France, 2012.

- Simon A., «Les enfants et la politique : contribution à l'étude des rapports ordinaire à la politique », Thèse de doctorat en science politique, Université de Montpellier, 2017.

- Sniderman P., «Les nouvelles perspectives de la recherche sur l'opinion publique », Politix, 1998, 41.

- Sniderman P. M., Brody R. A., Tetlock P. E., Reasoning and Choice. Exploration in Political Psycholgy, Cambridge University Press, 1991.

- Throssell K., Child and Nation; A Study of Political Socialisation and Banal Nationalism in France and England, P.I.E. Peter Lang, 2015.

- Tiberj V., «Compétence et repérage politiques en France et aux États-Unis : une contribution au modèle de "l'électeur raisonnant" ", Revue française de science politique, 2004, 54 (2).

- Tournier V., La politique en héritage ? Socialisation, famille et politique : bilan critique et analyse empirique, Thèse de science politique, IEP de Grenoble, 1997.

- Vaillancourt P. M., «Stability of Children's Survey Responses », Public Opinion Quarterly, 1973, 36.

- Zaller J., The nature and origins of mass opinion, Cambridge University Press, 1992. 